\title{
Gendering Border Studies? \\ Schnittstellen zwischen Border und Gender Studies
}

\section{Claudia Bruns}

\begin{abstract}
Territoriale Grenzen und Geschlechtergrenzen sind zunächst weitgehend getrennt voneinander erforscht worden. Standen in den Border Studies Staatsgrenzen im Mittelpunkt, befasste sich die Geschlechterforschung mit begrenzenden sozialen Rollenzuweisungen. Die Infragestellung binärer Oppositionen durch postmoderne wie postkoloniale Theorien ließ biologisch fundierte Geschlechtergrenzen wanken und machte Figuren des Dritten Raums, der Differenz bzw. der Mestiza Platz. Als sich damit auch der Begriff von Grenze in den Border Studies weg vom territorialen Paradigma den abstrakteren Dimensionen symbolischer Differenz öffnete, begann sich eine Schnittmenge zwischen Grenz- und Geschlechterforschung zu entfalten.
\end{abstract}

Schlagwörter

Gender Studies, Borders/Boundaries, Kollektiv/Körper, Postkolonialismus, Intersektionalität

\section{Einleitung: Schnittstellen zwischen Border und Gender Studies}

Bis in die 1990er Jahre hatte sich die Grenzforschung überwiegend auf zwischenstaatliche, militärische, koloniale und politisch-institutionelle Grenzen konzentriert. Erst mit der Rezeption postmoderner und poststrukturalistischer Theorien wurde aus der Grenze als Zweiheit die Grenze als abstrakte Relation. Die Prozesshaftigkeit des Grenzziehens wie auch die damit verbundenen (ambivalenten) Identitäts- und Differenzeffekte rückten allmählich in den Fokus der Border Studies (vgl. Paasi 1996; Newman 2006). Diese zunächst eher in den Literatur- und Kulturwissenschaften analysierten Dimensionen von Grenze, die dort als Figuren der Differenz oder Liminalität entfaltet wurden (vgl. Parr 2008), sollten bald auch in diejenigen Fächer eingehen, die sich traditionell mit politischen Grenzen befassten (wie Geschichts-, Politik-, Sozialwissenschaften oder die Geografie). Zur Überraschung mancher Literatur- und Kulturwissenschaftler*innen verabschiedete allen voran die Humangeografie den „realen Erdraum“ im Anschluss an die Integration konstruktivistischer Perspektiven weitgehend zugunsten des „geschaffenen Raumes“ (vgl. Lossau 2009, S. 36), sodass sich gesellschafts-, sozial- und kulturwissenschaftliche Perspektiven in den Border Studies aufeinander zubewegten.

Nicht zuletzt hat sich die transdisziplinäre Geschlechterforschung (vgl. Smith 1983; Breger 2010; Braun/Stephan 2013) eingehend mit den machtvollen Grenzen zwischen den Geschlechtern befasst wie auch mit Versuchen, diese zu subvertieren. Geschlechtlich codierte Dualismen wurden von feministischen Philosoph*innen als paradigmatische Struktur binärer Ordnung angesehen, als eine Vorlage für sämtliche Oppositionspaare, welche das abendländische Denken strukturieren (vgl. Klinger 1995). Die jüngeren Queer Studies zielen entsprechend darauf, die Allianz zwischen der Zweigeschlechterordnung und dem Regime der Heterosexualität, zwischen sex und gender, Begehren und Identität aufzubrechen und nach Formen der Repräsentation jenseits heteronormativer Dualismen zu suchen, nach Figuren und Räumen des Dritten (vgl. Johnson et al. 2005; Hall/Jgose 2012). 
Als sich, teils inspiriert von und teils parallel zu diesen Entwicklungen, der Begriff von Grenze in den Border Studies auffächerte und sich über das territoriale Paradigma hinaus den abstrakteren Dimensionen symbolischer Differenz öffnete, begann sich eine Schnittmenge zwischen Grenz- und Geschlechterforschung zu entfalten, deren Dimensionen und Verästelungen in verschiedensten Disziplinen kaum noch zu überblicken sind. Dennoch liegen bisher kaum Texte vor, die das transdisziplinäre Zusammenspiel zwischen Border und Gender Studies systematisch erfassen. Und - trotz Gloria E. Anzaldúas wegweisenden Arbeiten - ist die Integration geschlechtsspezifischer Perspektiven in die Border Studies keineswegs selbstverständlich geworden (vgl. Altink/Weedon 2010, ebenso Flores 2019).

Im Folgenden werden Entwicklungen des Grenzbegriffs in den Gender Studies skizziert, dann Tendenzen der feministischen Geografie beschrieben und einige wichtige Forschungsfelder benannt, die zentrale Schnittstellen zwischen der Geschlechterforschung und den Border Studies darstellen. Dazu gehören Fragen nach dem (Kollektiv-)Körper als Bindeglied zwischen Geschlechter- und Landesgrenzen und nach postkolonialen Perspektiven, welche den Schritt von binären räumlichen Ordnungen zu Räumen der Ambiguität anstoßen und damit Konzepte vom Dritten Raum als einem ebenso territorialen wie vergeschlechtlichten Differenzbereich verhandeln. Überdies werden methodologische Fragen der Medialität, Performativität und Intersektionalität berührt, welche für Prozesse des bordering von Gender Studies und die Integration räumlicher Kategorien in die Geschlechterforschung relevant sind. Abschließend sollen Definitionen des Begriffs von Grenze in den Border Studies skizziert werden, welche symbolische Grenzen bzw. Figuren der Differenz in die Konzeption von physisch-territorialen Grenzen einbeziehen und damit die Border Studies eng an die Gender Studies heranführen. Im Fazit werden aktuelle Forschungsfragen benannt, die beide Wissensgebiete miteinander verbinden und füreinander interessant werden lassen.

\section{Geschlechter/Grenzen in den Gender Studies}

Das Verständnis von Grenze hat sich nicht nur in den Border Studies allmählich von einer physisch-materiellen Realität zu einer symbolisch-sozialen Figuration der Differenz bewegt und Fragen nach dem Zusammenspiel von materiellen, institutionellen und symbolischen Prozessen ausgelöst. Innerhalb der Geschlechterforschung hat es ähnliche Transformationsprozesse in Bezug auf die Konzeption der Grenze zwischen den Geschlechtern gegeben.

Hatte Simone de Beauvoir mit Le deuxième sexe (1949) schon früh darauf hingewiesen, dass niemand ,als Frau geboren', sondern ,dazu gemacht' werde, griffen Frauen in den 1960er und 1970er Jahren auf ihre Analysen zurück und übten Kritik an ungleichen, polaren ,Geschlechterrollen' die gesellschaftlich bedingt und keineswegs natürlich seien. Die Kategorie Geschlecht wurde als „sozialer Platzanweiser“ verstanden, der Frauen und Männern ihren „Ort in der Gesellschaft, Status, ihre Funktionen und Lebenschancen“ (Knapp 1988, S. 12) zuweise. Weltweit forderten Frauen, diese räumlich-soziale Ordnung zu durchbrechen und zugleich eigene Frauen- und Lesben-Räume zu schaffen, die als „Möglichkeitsbedingung von Identitätsformationen“ (Runte 2006, S. 10) entdeckt wurden.

In der Folge inspirierten sich soziale Bewegung und akademische Theorieentwicklung wechselseitig. Die Frauenforschung konzentrierte sich anfänglich auf Analysen gesellschaftlicher Machtungleichheit und kritisierte den Ausschluss weiblicher Lebens- und Erfahrungsräume als Forschungsgegenstand wie von Frauen als Forschenden aus der Wissenschaft (vgl. Scott 
1986; Hausen/Wunder 1992). In der Folge wurde die Grenze zwischen den Geschlechtern zunehmend ihrer Natürlichkeit entkleidet. Die Wissensproduktion selbst rückte in den Fokus der Geschlechterforschung, allumfassende Objektivitätsansprüche sahen sich durch das Konzept des „situierten Wissens“ (Haraway 1995b) zurückgewiesen und an einen bestimmten Ort, einen notwendig partialen Blick zurückgebunden.

Zuvor hatten Feministinnen of colour in den USA ihre eigene Unsichtbarkeit innerhalb der weiß dominierten bürgerlichen Frauenbewegung wie -forschung kritisiert und bereits in den 1970er Jahren intersektionale Analysen eingefordert, welche die Beziehung zwischen mehreren, gleichzeitig vorhandenen Achsen der Diskriminierung (race, class, gender) in den Blick nehmen sollten. Trennende Grenzen schienen nicht mehr nur zwischen den zwei Geschlechtern zu verlaufen, sondern auch innerhalb der Kategorie Frau selbst. Die Achsen der Differenz mussten komplexer und multidimensionaler gedacht werden. Prominent seien hier für intersektionale Ansätze aus dem angloamerikanischen Raum das Combahee River Collective (1982), bell hooks, Alice Walker, Kimberlé Crenshaw (1998) und Patricia Hill Collins (2019) genannt.

Ende der 1980er Jahre trat Männlichkeit als eigenständige Analysekategorie stärker hervor, was den Wandel von der Frauen- zur Geschlechterforschung einleitete. ${ }^{1}$ Angeregt durch poststrukturalistische Theorien entwickelte sich eine feministische Kritik an der bis dahin gültigen Trennlinie zwischen sozialem und biologisch-sexuellem Geschlecht. Aus (radikal-)konstruktivistischer Perspektive wurde im Anschluss an die amerikanische Philosophin Judith Butler das „biologische Geschlecht“ als performativer Effekt diskursiver Macht- und Aushandlungsprozesse verstanden, der - im Rekurs auf Jacques Derridas Konzept differenzieller Iteration einer permanenten Wiederholung zur Verfestigung der „zwangsheterosexuellen Matrix“ (Butler 1990) bedürfe. Butlers Werk löste die sogenannte Sex/Gender-Debatte über das Verhältnis zwischen Körper, Materie und Diskurs aus (vgl. Waniek/Stoller 2001).

In (Gegen-)Reaktion auf Positionen des radikalen Konstruktivismus nahm die Körpergeschichte einen Aufschwung, etwas später die Emotionsgeschichte sowie neue phänomenologische, kognitionstheoretische oder psychoanalytische Konzepte des Prädiskursiven (vgl. List 1997). Postkonstruktivistisch wurde Materie im New Materialism nicht mehr als „stumme Verfügungsmasse und einfaches Objekt menschlichen Zugriffs“ (Hoppe/Lemke 2015, S. 262) angesehen, sondern in Bezug auf ihr Potenzial zur menschenunabhängigen Selbstorganisation und ihre bisher unterschätzte Eigenmacht. Natur und Kultur sollten nicht mehr als Gegensätze, sondern als ineinander übergehend begriffen werden (vgl. Haraway 1995a; Bennett 2004; Barad 2007). Entsprechend verstand Butler die

„Geschlechterdifferenz [als] ein[en] Ort, an dem wieder und wieder eine Frage in Bezug auf das Verhältnis des Biologischen zum Kulturellen [...] gestellt werden muss [...]. Wenn wir sie als eine Grenzvorstellung verstehen, so hat die Geschlechterdifferenz psychische, somatische und soziale Dimensionen, die sich niemals gänzlich ineinander überführen lassen [...]“ (Butler 1997, S. 35).

In dem Maße, wie die klare Grenze zwischen natürlichem und kulturellem Geschlecht ins Wanken geriet, wurde sie in der Geschlechterforschung selbst zum Gegenstand der Reflexion. Die Grenze zwischen den Geschlechtern könne auch, so die Literaturwissenschaftlerin Anette

1 Der Soziologe Pierre Bourdieu stellte prominent einen Bezug zwischen Männlichkeits- und Raumordnung her (Bourdieu 1997, S. 2; vgl. Lossau/Lippuner 2004). Zuvor waren bereits in den Geschichtswissenschaften und der Kunstgeschichte Beiträge zur Beziehung zwischen geschlechtlicher und räumlicher Ordnung entstanden. 
Runte im Anschluss an Niklas Luhmann, als „binäre Struktur“ mit zwei Polen verstanden werden, zwischen denen sich eine „unendliche Oszillation“ vollziehe, die nur durch eine gewisse Asymmetrie, eine „winzige Präferenz“ (Runte 2006, S. 9) für eine der beiden Seiten, zum Stillstand gebracht werden könne. In dieser Stilllegung liege die „Entscheidung dieser Logik für den Mann“ (ebd.). Dennoch, so heben dekonstruktivistische Lektüren im Anschluss an Derrida hervor, erweisen sich beide Pole letztlich als voneinander abhängig. Mit diesem Perspektivwechsel in Richtung Relationalität von Geschlechterverhältnissen verloren die Grenzen zwischen den Geschlechtern nicht nur ihre biologische Selbstverständlichkeit, sondern auch ihre ausschließlich trennende Funktion. Vielmehr wurden Männlichkeit und Weiblichkeit als füreinander konstitutive Einheiten entworfen (Runte 2006, S. 9).

Inzwischen ist die sämtliche Felder des Wissens durchziehende Geschlechtergrenze selbst zu einer „schwankenden Grenze“ (Butler 1997, S. 35) geworden, die feste Identitäten auflöst und Begehrensstrukturen verflüssigt. Dekonstruktivistische Lesarten geschlechtlicher Identitäten werden gegenwärtig mit deren „alltagsweltlicher Nivellierung und scheinbar unbegrenztem Fluktuieren“ (Runte 2006, S. 14) assoziiert; setzt doch die (Post-)Moderne auf eine Bewegung permanenter Grenzüberschreitung, eine Art „reflexiv gesteigerte Ambivalenz“ (ebd.), auf welche Figuren der Spaltung, der Vermischung und Identitätsauflösung projiziert werden.

In der Folge wurden auch Phänomene der geschlechtlichen Grenzüberschreitung, also der Travestie, Androgynie oder Transgeschlechtlichkeit eingehender untersucht und in Beziehung zu bestimmten Grenzorten (besonders prominent für Tijuana) oder raumkonstituierenden performativen Praktiken gesetzt (vgl. etwa Cotten 2012). Die Figur des Cyborgs rüttelte überdies an den im abendländischen Denken fest verankerten Grenzen des Menschen zum Posthumanen, zu Tieren und Dingen (vgl. Haraway 1995a; Mateos-Aparicio 2007). Es geht um „conative bodies“, die in „Assemblagen“ (Bennett 2004, S. 366) miteinander verbunden sind, und um ein Werden als „Involution“ (Deleuze/Guattari 1992, S. 399f.), das statt einer Abstammungsund Grenzlinie mit Anfangs- und Endpunkt eine „Zone der Nachbarschaft“, einen Bereich der Ununterscheidbarkeit denken lässt, wodurch Ereignisse an der Grenze auf neue Weise theoretisiert werden können, etwa als bordercrossings (Kuster 2018), und sich einer queeren Geografie öffnen (vgl. z.B. Lau et al. 2013).

Grenzen wurden bald auch in der historischen Transferforschung (vgl. Pratt 1991; Somerville/Perkins 2003; Werner/Zimmermann 2003) und dann auch in den Border Studies nicht mehr als fix, sondern als fluide und relationale Zonen des Kulturkontakts entdeckt. Insofern lassen sich bestimmte Parallelen und Berührungspunkte zwischen Gender und Border Studies in Bezug auf ihr jeweiliges Verständnis von Grenze ausmachen.

\section{Geschlechterperspektiven in den Border Studies}

Die für die Gender Studies typischen theoretischen Brüche und Paradigmenwechsel finden sich in den Border Studies wieder, allen voran in der feministischen Geografie: Anfänglich sollten Frauen erst einmal sichtbar gemacht werden und geografische Traditionen wie die der Länderkunde, z.B. durch Reiseberichte von Frauen, uminterpretiert werden (vgl. Women and Geography Study Group 1997, S. 56f.). In den 1970er Jahren rückten ökonomische Ungleichheiten und sog. ,patriarchale' Strukturen in den Fokus, verbunden mit der Frage, „inwiefern spezifische geografische Orte unterschiedliche Geschlechterrollen und Geschlechterbeziehungen konstituieren“ (Fredrich 2012, S. 32). Schließlich kam es in den späten 1980er Jahren 
im Anschluss an postmoderne Theorieentwicklungen zu einer kritischen Reflexion der Kategorie Frau und zur Herausbildung von „Geografien der Differenz“ (Women and Geography Study Group 1997, S. 76), die zu einer Subvertierung von dualistischen Kategorienbildungen anregten. In diesem Zuge rückten auch andere konstitutive Differenzen in den Blick, etwa die Verflechtung von race, class, gender und sex mit Kategorien von place/space (vgl. Wastl-Walter 2010, S. 33-36; Strüver 2005).

Über die feministische Geografie hinaus etablierten sich allmählich geschlechtertheoretische Perspektiven in fast allen Disziplinen, die sich mit Grenzen befassen. Dennoch bezogen die Border Studies im Allgemeinen (gemessen an der Zahl der Zeitschriften, die sich thematisch damit befassen, Anthologien und Forschungsthemen in Institutionen) die Kategorie Geschlecht erst relativ spät und bis heute eher zögerlich in ihre Analysen ein. So kommen die meisten Beiträge, welche die gegenwärtigen Herausforderungen der Border Studies, wie etwa den Begriff der Grenze zu definieren versuchen, ohne oder mit marginalem Bezug zur Kategorie Geschlecht aus - und das, obwohl Anzaldúa (1987) mit ihrer Monografie zur US-mexikanischen Grenze einen anderen Weg eingeschlagen hatte. Ihr postmoderner Blick auf borderlands als Räume des Übergangs, der Verflechtung und intersektionaler ,Vermischung' wurde zwar weltweit rezipiert, aber nur von Teilen der Grenzforschung aufgegriffen - vorrangig dort, wo es um literatur- und kulturwissenschaftliche Perspektiven auf die Lebens- und Erfahrungsräume von Frauen of colour bzw. des Chicana Movement und um die Grenze zwischen den USA und Mexiko geht (vgl. Fellner in diesem Band).

\subsection{Postkolonialer Feminismus}

Postkolonial inspirierte Analysen haben in ihrer feministischen Spielart (vgl. Spivak 1988; Blunt/Rose 1994; Lewis/Mills 2003) dagegen früh geschlechtsspezifische Perspektiven integriert, meist im Kontext kritischer Kolonialgeschichts- oder Okzidentalismusforschung (vgl. Dietze 2006). Grenzen wurden hier als Verschränkungen von räumlichen mit (kolonial-)rassistischen und geschlechtlichen Differenzkategorien verhandelt. So lösten die Arbeiten des gebürtigen Palästinensers Edward Said (1978/2009) eine Reflexion der (Selbst-)Entwürfe des Okzidents aus, welche über die Abgrenzung vom Orient als dem effeminierten, erotisierten und exterritorialisierten Anderen erfolgt sei. Sein Ansatz inspirierte überdies Untersuchungen zur ,Erfindung‘ eines gegenüber dem Westen abgewerteten Osteuropa als einem middle ground zwischen männlicher imaginierter ,Zivilisation' und weiblich codierter ,Wildheit', etwa durch Larry Wolff und Marija N. Todorova. Aber auch die Grenzziehung gegenüber dem Islam in der Debatte um Islamismus, Schleierverbot und die Vereinnahmung emanzipatorischer Positionen für die europäisch-westliche, Wir'-Gruppe schloss an Saids Thesen an (vgl. Braun/ Mathes 2007; Dietze 2019).

Binäre Gegenüberstellungen im (post-)kolonialen Kontext zu unterlaufen war auch ein zentrales Anliegen der kulturtheoretischen Arbeiten Homi K. Bhabhas. Die Struktur symbolischer Repräsentation selbst lasse einen Dritten Raum entstehen, denn, um Bedeutung zu produzieren, müsse sich das „,in der Aussage festgelegte Ich und Du“ fortgesetzt bewegen - eine Bewegung, in der "diese beiden Orte [...] einen Dritten Raum durchlaufen“ (Bhabha 2007, S. 55). Dadurch entstehe notwendigerweise eine Ambivalenz im Akt jeglicher Interpretation, die es gegen die Autorität westlicher Kategorien und Einheiten zu setzen gelte (ebd., S. 58). Der Dritte 
Raum der Hybridität ist Bhabha zufolge immer schon ein „Schwellen- und Übergangsraum“, der durch seine „Grenzlage“, ein „Überlappen und „De-Platzieren von Differenzbereichen“ charakterisiert ist (ebd., S. 2, 5, 9).

Bhabhas Ansätze wurden von feministischen Studien vielfach aufgegriffen. Vor allem Feministinnen of colour gaben den Anstoß dazu, die Kritik an herkömmlichen Binarismen mit eurozentrismuskritischen Perspektiven zu verbinden. Nicht zuletzt zeigte sich Anzaldúa durch Bhabhas Konzept vom Dritten Raum inspiriert (vgl. Rutherford/Bhabha 1990). Der Figur der mestizischen Frau, die aus einem Prozess von verschiedenen kulturellen wie territorialen Grenzziehungen hervorgehend entwickelt wird, schreibt Anzaldúa das Potenzial zu, binäre Strukturen aufbrechen zu können, weil sie in besonderer Weise Ambiguität repräsentiere (vgl. Anzaldúa 1987, S. 21). Mit ihrer autobiografisch-poetischen Art zu schreiben sprengte Anzaldúa Genregrenzen, forderte heterosexuelle Normen der Chicano/a-Kultur wie auch Rassismen der US-amerikanischen (Re-)Kolonialisierung heraus (vgl. Bandau 2004; Heide 2004; Sieber 2004; Perales 2013). Borderlands sollten Anzaldúa zufolge nicht nur als physisch-territoriale, sondern auch metaphorische Räume erfasst werden, da symbolische Geschlechter- und ,Rassengrenzen' machtvoll in sie eingeschrieben seien.

Während die von Anzaldúa konstatierte enge Verflochtenheit von physischen und symbolischen Grenzen in den Border Studies erst deutlich später zum Thema wurde, ist die Konnotation von Frauen mit Grenzen und Grenzziehungsprozessen zu einem wichtigen Forschungsgegenstand geworden, der vor allem im US-amerikanischen Kontext, aber auch zur Ermächtigung von (Frauen-)Bewegungen des Globalen Südens und in postkolonialen Studien aufgegriffen werden sollte (vgl. Lugones 1992; Saldívar-Hull 2000; Castillo/Tabuenca Córdoba 2002; Castañeda et al. 2007; Keating 2016). Im Anschluss an Anzaldúa entstand in den 1980er-Jahren ein Border oder Mestiza Feminism, der radikale Kritik an akademischer Wissensproduktion, vergeschlechtlichten und rassisierten Identitätspolitiken und der Marginalisierung migrantischer Leben übte (vgl. Kron 2011, S. 202).

In den Literatur- und Kulturwissenschaften erlebten Forschungen zu Grenzen als Schwellenräume, Zonen des Übergangs und der Liminalität um die Jahrtausendwende einen Aufschwung, die sich für Interdiskurse, das Subnormale oder die Spalte interessieren (vgl. Parr 2008). Androgynie wurde zur liminalen Figur par excellence, die Grenze „von gender und Kultur bis hin zur Beschreibung der Lichtwellen“ zu einer „Metapher der Metaphern“, die „überkommene Konstrukte der Identität“ (Saul/Möbus 1999, S. 10, Herv. i. O.) zugunsten pluralistischen Denkens zur Disposition stellte. Insbesondere Begriffspersonen wie der Mestiza oder dem Cyborg wurde zugeschrieben, kategoriale Grenzen sowohl zu überschreiten als auch infrage stellen zu können (vgl. Mertlitsch 2016, S. 223).

\subsection{Migration, Gewalt und Territorialität in der Grenzforschung}

In der kritischen Migrations-, der ethnologischen Urbanitäts- und der sozialwissenschaftlichen Forschung wurde die Kategorie Geschlecht ebenfalls zu einer wichtigen Dimension der Analyse (vgl. Lutz 2008), wenn auch unterschiedlich eng an die Border Studies anschließend. Zahlreiche Untersuchungen befassten sich damit, wie sich nationale oder kontinentale (territorial-juridische) Praktiken der Grenzziehung auf das Leben von Frauen und Männern an der 
Grenze oder bei alltäglichen wie auch irregulären Grenzüberschreitungen auswirkten (vgl. z.B. Morokvasic 2006; Akyüz 2017; vgl. auch Leutloff-Grandits in diesem Band).

Ein herausragendes Thema war das der sexualisierten, geschlechtsspezifischen Gewalt, die Frauen und Männer auf der Flucht, vor allem bei der Überquerung der US-mexikanischen oder europäischen Außengrenze, trifft oder wenn sie in Lagern festgehalten werden, in denen Menschenrechte oftmals systematisch ausgesetzt sind (vgl. Ticktin 2008; Wright 2011; Del Re 2018; Hernández 2019; Tyszler 2019).

Galten in der Migrationsforschung Männer lange als prototypische Migrierende, rückten Frauen zunächst im Kontext von Familienmigration, dann aber auch in den Bereichen von grenzüberschreitendem Menschenhandel (vgl. Lobasz 2009), Sex Trafficking (vgl. z.B. Penttinen 2008; Segrave 2012; Vuolajärvi 2015) oder im Kontext von Heirats- oder Arbeitsmigration für Haushalts- und Pflegetätigkeiten (vgl. Wastl-Walter 2010, S. 112-120; z.B. Toksöz/Ünlütürk Ulutaş 2012) in den Blick. Der sich weltweit erhöhende Anteil migrierender Frauen wurde als ,Feminisierung der Migration“ diskutiert (Le Breton 1998; Spindler 2011). Die zunächst überwiegend männliche Repräsentation von Migration in den öffentlichen Medien differenzierte sich nach dem ,Sommer der Migration“ (Hess et al. 2016; Sehnbruch 2019). Praktiken des Widerstands, counter narratives (Bhabha), diasporic narratives (Gilroy 1993), die Eigenmächtigkeit von Migrierenden bzw. die ,Autonomie der Migration‘ (Römhild 2009; Pieper et al. 2014) rückten in den Blick.

Diese verschiedenen Forschungen zu Geschlecht und Grenze bilanzierend, kritisierten die britischen Kulturhistorikerinnen Henrice Altink und Chris Weedon, dass viele der aktuellen Studien vorrangig gegenwartsbezogen seien, sich im Kontext von Migrationsstudien verorteten und Grenzen im Sinne von nationalstaatlichen Grenzregimen verstünden. Auch tendiere die Grenzforschung dazu, die Kategorie Gender mit Frauen gleichzusetzen, oder ignoriere weitgehend die Intersektionen mit anderen Differenzkategorien wie ,Rassec, Ethnizität oder Klasse (vgl. Altink/Weedon 2010, S. 9). Sie forderten, Genderkonstruktionen nicht nur als rein metaphorische Grenzkonstruktion zu untersuchen, sondern diese in Relation zu territorialen Grenzziehungs- und Repräsentationsprozessen zu setzen und das jeweilige Zusammenspiel auf seine materiellen wie symbolischen und politischen Effekte hin zu befragen. Es müsse darum gehen, zu erforschen, wie borderlands einerseits hegemoniale Geschlechterformationen herausforderten und andererseits selbst zur Herausbildung von Genderideologien beitrügen (vgl. ebd., S. 2).

Der Anspruch von Aaron, Altink und Weedon, mit ihrem Band Gendering Border Studies (2010) ein neues Feld zu erschließen, wird durch die rege Forschung zu „American Studies as Border Studies“ im Kontext des Chicanola Movement relativiert (Ganser 2013, S. 510). So hatte etwa Melissa W. Wright (1998) Anzaldúas Ansatz weiterentwickelt, indem sie am Beispiel von Montagebetrieben des Niedriglohnsektors in der US-mexikanischen Grenzregion der Frage nachging, wie Klassendifferenzen in die Formation von vergeschlechtlichten und nationalisierten Subjekten hineinspielten. Lionel Cantús Arbeiten zur „Sexualität der Migration“ mit einem Fokus auf nach Los Angeles migrierte mexikanische Männer und der vergeschlechtlichten Migrationspolitik staatlicher Behörden hatten der Intersektion von Geschlecht und Grenze ebenfalls starke Impulse verliehen (vgl. Cantú 2009). Dennoch verweist der Band von Aaron, Altink und Weedon (2010) - vor allem für den europäischen Kontext - auf erhebliche Desiderate im Schnittfeld von Border und Gender Studies. 
So lässt sich festhalten, dass Grenzen in der europäischen Forschung meist entweder in territorial-politischer oder symbolisch-differenztheoretischer Hinsicht untersucht worden sind; selten in einer Zusammenführung beider Perspektiven. Insbesondere moderne staatliche Grenzziehungsprozesse sind kaum aus geschlechtertheoretischer Perspektive analysiert worden (Reichert 2011). Zwar befasste sich kulturgeografische Forschung mit dem ,Mapping von Sexualitäten“ im sozialen Raum, Landesgrenzen spielten dabei jedoch weniger eine Rolle (vgl. Bell/ Valentine 1995; Krewani 2000; Browne et al. 2009). Dies hat dazu geführt, dass einige der politisch einflussreichsten Grenzziehungsdiskurse der Gegenwart rund um den Ost-West-Konflikt und die Berliner Mauer lange als geschlechtsneutrale Exklusions- und Inklusionsformen wahrgenommen wurden (vgl. Reichert 2011, S. 35) - und das, obgleich sich an Landesund Zollgrenzen „unterschiedliche Diskriminierungsformen“ (ebd., S. 36) in besonderer Weise überlagern.

Dieses Desiderat gilt auch für die europäische Außengrenze, der sich relativ wenige kulturoder medienwissenschaftliche Arbeiten aus geschlechtertheoretischer Perspektive widmen. Eine Ausnahme macht etwa die Kulturwissenschaftlerin Francesca Falk (2011), die Grenzbilder Europas anhand von Fotografien von Bootsflüchtlingen auf ihre geschlechtlichen, kulturhistorischen und medialen Codierungen hin untersucht. Sie zeigt, dass sich die Spuren unseres Bildgedächtnisses von Immigrierenden als gefährliche Infektionsherde bis in die Frühe Neuzeit zurückverfolgen lassen. Auch Philipp Sarasin (2004) analysiert die problematische Metaphorisierung von (muslimischen) Terroristen als ,Infektionsgefahren' in Zeiten grenzüberschreitender Globalisierung. Eine kulturwissenschaftliche Perspektive auf europäische Grenzen, die explizit geschlechtliche und rassismuskritische Analysedimensionen einbezieht, wird im Band Europas Außengrenzen. Interrelationen von Raum, Geschlecht und „Rasse“ entfaltet (vgl. Gradinari et al. 2020).

\subsection{Medialisierungen, verschlechtlichter' Grenzen}

Um Grenzen zu legitimieren, müssen sie in spezifischen Kommunikationsräumen plausibilisiert werden. Daraus folgt, dass eine feministische Perspektive auf (physische) Grenzen reflexiv in Bezug auf das Medium sein sollte, das die Grenzbilder, -techniken oder -architekturen übermittelt (vgl. Reichert 2011, S. 36). So lässt sich etwa in deutschen ,Mauerfilmen` eine enge Verbindung zwischen Grenzdarstellungen und binären Geschlechtercodes ausmachen, welche physische Grenzziehungen unterstützten und legitimierten (vgl. ebd., S. 41). In verschiedenen medialen Figurationen von Grenze reproduzierten sich somit Grenzsetzungen „als performative und soziale Praxis der Herstellung von Geschlecht“ (ebd.) und tragen auf diese Weise zu Geschlechterasymmetrien bei.

Die Kulturwissenschaftlerin und Filmemacherin Brigitta Kuster widmet sich in ihrer Monografie Grenze filmen (2018) vor allem den audiovisuellen Gegenbildern und Widerstandspraktiken, die Grenzüberschreitungen hervorbringen und begleiten. Das „Kino der Migration“ habe die „Bewohnbarkeit von Zwischenräumen, Zeit-Räumen der Verbindung oder rites de passages zum Ziel“ (ebd., S. 203). Das Verhältnis zwischen dem Potenzial des Bildes und den migrantischen Bewegungen sowie die Interferenzen, die deren wissenschaftliche Bearbeitung erzeugt, stehen dabei im Fokus. 
Auch in den neueren Security Studies findet sich die Einbeziehung geschlechtlicher Perspektiven. Paradigmatisch ist etwa die Analyse von Überwachungsbildern an der Grenze. Das Bild eines vermeintlich fremden und gefährlichen, männlich codierten (Gegen-)Kollektivs wird zum Beispiel durch die Art der verdichtenden Darstellung in Nacht- und Zeitrafferaufnahmen von Grenzanlagen in Ceuta und Mellila verstärkt. Die Aufnahmen lassen ,die Anderen ' als massenhaft, hemmungslos und unaufhaltsam die Grenze überquerend erscheinen, also als „bedrohliche Masse“ oder „anonyme Flut“ (Reichert 2011, S. 49). Maßnahmen zur Grenzsicherung werden damit indirekt als legitim ausgestellt (ebd.). Eine Reihe von Arbeiten befasst sich aus geschlechter- und rassismuskritischer Perspektive mit biometrischer Datenerzeugung, Algorithmengenerierung und anderen Formen der Technologisierung und Digitalisierung von Grenzen (vgl. Magnet 2011; Koskela 2012; Côté-Boucher 2020).

Die Verbindung zwischen Grenzforschung und feministischer Theorie stellt trotz allem noch immer eine Herausforderung dar. Die US-amerikanische Kommunikationswissenschaftlerin Lisa A. Flores rief noch 2019 zur Entwicklung einer Feminist Border Theory auf. Die Verflechtung zwischen den Wissensfeldern von Border und Gender Studies bedürfe, so Flores, einer intersektionalen Herangehensweise (vgl. auch Schäfer in diesem Band). Grenzen sollen als geopolitische boundaries verstanden und zugleich auf diese Grenzziehungen nicht begrenzt, sondern ebenso als Effekt von Figuren der Differenz wie auch - im Anlehnung an Anzaldúa als Orte der Intervention gefasst werden (vgl. Flores 2019, S. 113, 115).

\section{Mapping the body: Interrelationen zwischen Körper- und Landesgrenzen}

Einer der zentralen Schnittpunkte zwischen territorialen Grenzziehungsprozessen und geschlechtlichen Differenzkonstruktionen lässt sich in der engen metaphorischen Beziehung ausmachen, die zwischen Landeskörper und menschlichem Körper hergestellt wurde. Zum einen werden physisch-territoriale Räume im bildhaften Symbolsystem der Karte als Landeskörper beschrieben, zum anderen repräsentieren Erdteilpersonifikationen bestimmte kollektive Räume allegorisch, wie die Verkörperungen der Kontinente oder Nationen durch Frauenfiguren in der Frühen Neuzeit oder durch biblische Figuren wie die Heiligen Drei Könige oder die Söhne Noahs im Mittelalter (vgl. Bruns 2009 sowie i.E.). Auf diese Weise symbolisiert der allegorische Körper kollektive wie soziale Räume und zieht Grenzen, die geschlechtlich codiert sind. Die Vorstellung vom Kollektivkörper geht indes über eine rein metaphorische Funktion hinaus, indem sie materielle politische und institutionelle Effekte, etwa im Zuge der Nationenbildung, generiert (vgl. Koschorke et al. 2007, S. 11).

\subsection{Allegorie, Kartografie und Frontiermythos}

Aus der Frage nach den geschlechtlichen Codierungen des nationalen Kollektivkörpers entwickelte sich ein spezifisches Interesse an dem Zusammenhang zwischen Geschlecht und Grenze (vgl. Abrams/Hunt 2000, S. 191). Frauenfiguren repräsentierten allegorisch die Einheit wie auch die Grenzen der Nation (ebd., S. 193; vgl. Wenk 1996; Wintle 2009). Als „Grenzwächterinnen“ (Domosh/Seager 2001, S. 160f.; vgl. auch Michels 2009) kam ihnen die Aufgabe zu, die Demarkationslinie zwischen Kollektiven, ,Rassen“ und Geschlechtern aufrechtzuerhalten. Das Weibliche wurde an der symbolischen Schnittstelle zwischen „einem zu ähnlichen 
Anderen [...] und einem radikal unähnlichen Anderen“, „zwischen Identität und Alterität“ (Funk 1998, S. 200) verortet. Schon in den populären Reiseromanen Jean de Mandevilles Mitte des 14. Jahrhunderts finden sich mythische Amazonen als geschlechtliche Mischwesen mit männlich-kriegerischen Eigenschaften, die der Grenzregion zwischen Europa und Asien zugewiesen wurden, wo sie die Christenheit Europas gegen apokalyptische Feinde verteidigen sollten (vgl. Mandeville 2005, S. 165f.). Und auch die alttestamentliche Figur der Eva verweist auf der Ebstorfer Weltkarte nicht nur auf die moraltheologische (vergeschlechtlichte) Grenze zwischen Gut und Böse, sondern auch auf die transzendente Grenze zwischen diesseitiger und jenseitiger Welt, die in den Karten als das Paradies umgebende physische Mauer visualisiert wird (vgl. Bruns i.E.).

Die Karte besitzt für die historische Analyse von vergeschlechtlichten (Grenz-)Räumen eine besondere Erkenntnisqualität (vgl. Schlögel 2007, S. 46f.). Mit John Brian Harley lassen sich Karten als „mit Bedeutung aufgeladene Bilder“ (Harley 1988, S. 277) verstehen, die als Teil der Wissensdiskurse und Imaginationen einer Gesellschaft untrennbar mit Machtverhältnissen verbunden sind. Durch die Art ihrer Zeichen-, Symbol- und Farbwahl tragen sie in ihrer eigenen Bild- und Formsprache dazu bei, Weltwahrnehmung zu strukturieren und eine besondere Form von Evidenz zu erzeugen (vgl. Pápay 2005, S. 90). Erst mit der kartografischen Darstellung Europas als kolonialer Königin zu Beginn der Frühen Neuzeit markierten z.B. die linear gezeichneten Körpergrenzen der weiblichen Figur auch die geografisch-territorialen Grenzen des Kollektivkörpers Europa, und zwar lange bevor man nationalstaatliche Grenzen linear einzuzeichnen begann (vgl. Schmale 2000; ebenso Wintle 2009).

Die symbolischen Zuweisungen an Frauenkörper, die Scheidelinie zwischen verschiedenen Kollektiven zu repräsentieren, hatten ambivalente Effekte für Frauen (vgl. Yuval-Davis 2001, S. 81). Diese erhielten einerseits die Möglichkeit, ihre politische und soziale Bedeutung zu stärken, sei es im Kontext der deutschen 1848er-Bewegung oder im Einsatz an der Ostgrenze während der NS-Zeit (vgl. Harvey 2010). Auch im kolonialen Kontext konnten weiße Frauen von der ihnen zugeschriebenen Rolle als ,Hüterinnen kultureller Grenzen` durchaus profitieren (vgl. Mamozei 1992; Michels 2009; Dietrich 2015).

Andererseits sahen sich Frauen durch ihre allegorische Funktion einem starken Druck zu Konformität ausgesetzt, denn die Grenze materialisiert sich in genau jenen Körpern, die sie überschreiten, sie „schreibt sich in das Fleisch ein, wird auf dem Rücken getragen“ (Weier et al. 2018, S. 74). Ein Abweichen von der normativen Geschlechterrolle gefährdete besonders in Krisenzeiten die Nation als Ganzes, wie sich am Beispiel des ,Amiliebchens‘ zeigen lässt (vgl. Nieden 2002). Damit verstärkte sich für Frauen auch die Gefahr, in kriegerischen Auseinandersetzungen Opfer von Vergewaltigungen (vgl. Abrams/Hunt 2000, S. 195) - so in besonders extremer Form während des Jugoslawienkriegs und während des Genozids in Ruanda (vgl. z.B. Jones 2002; Crawford 2017) - oder von Menschenhandel und Zwangsprostitution zu werden. Für indigene Frauen waren Grenzzonen oft von sexualisierter Gewalt, Vertreibungen, ökonomischer Ausbeutung, zerstörten Familienbanden oder den Zumutungen rassistisch-sexistischer Stereotypisierungen durch die Kolonisierenden geprägt, sie konnten aber auch widerständische Praktiken und transkulturelle Dynamiken evozieren (vgl. z.B. Chaudhuri 1998; Clancy-Smith 2005; Baker 2007).

Männlichkeitsideale trugen auf eigene Weise zur Nationenbildung und ihren Abspaltungen bei. So zeigen aktuelle Analysen der Politischen Geografie, etwa zum US-amerikanischen Kampf gegen den Terror, dass dieser mit bestimmten, aus dem Frontiermythos entlehnten Männlich- 
keitsvorstellungen verbunden war (vgl. Hannah 2005, S. 553). Dieser Mythos stand für den unbedingten Willen, die Westgrenze gewaltsam stetig weiter gen Osten zu verschieben (Turner 1920; vgl. Schetter/Müller-Koné in diesem Band). Die Überwindung der als weiblich und primitiv codierten ,Naturgrenze“ durch männlich konnotierte Kräfte des ,Fortschritts` entwickelte sich zu einer paradigmatischen (Grenz-)Figur der US-amerikanischen Geschichte, die bis heute kriegerische wie sexualisierte Gewalt durch den Bezug auf eine besondere ,Zivilisierungsmission` rechtfertigt (vgl. Hogan/Pursell 2008; Slotin 1998).

\subsection{Border Thinking als, verkörpertes Bewusstsein' des (De-)Kolonialen}

In dem Maße wie der Körper ins Zentrum der Analysen rückte, stellte die feministische politische Geografie ihrerseits ein zentrales, Grenzen konstituierendes, räumliches Ordnungsmuster der Geografie infrage: die Einteilung der Welt in verschiedene Skalen wie lokal, national und global (vgl. Fredrich 2012, S. 44). Solche Skalierungen seien entlang von Dichotomien organisiert, die das Globale dem Lokalen überordneten, was den Ausschluss von weiblichen Lebenszusammenhängen aus wissenschaftlichen Fragestellungen verstärkt habe (vgl. ebd., S. 48). Künftig müsse es eher um die Wechselbeziehungen zwischen den Skalen gehen (vgl. Marston et al. 2000).

Überhaupt sind Räume, Doreen Massey zufolge, gerade nicht als hierarchisch angeordnete, abgeschlossene Einheiten zu verstehen, sondern als zufällige, in Beziehung miteinander stehende Arrangements vielfältiger Beziehungen (vgl. Massey 1999, S. 283). Entsprechend kritisch verhandelt die feministische Geografie zentrale kolonialistische Masternarrative der Moderne wie ,Evolution` und ,Fortschritt', welche die Zeit verräumlichen, indem sie die Welt in Zonen höherer oder niedrigerer ,Entwicklungsstufen` einteilen und ,Anderen` den Ort der Rückständigkeit zuweisen (ebd., S. 280f.).

Ansätze des dekolonialen Feminismus spitzen diese Kritik zu, indem sie hervorheben, dass der Kolonialismus die Moderne überhaupt erst hervorgebracht hat, womit sie u.a. an die Arbeiten von Anzaldúa, Walter Mignolo, Catherine Walsh, Anibal Quijano und Rolando Vázquez anknüpfen (vgl. Lugones 2010; Icaza 2017). In der Konsequenz müssten hegemoniale Geschichtserzählungen, welche die Moderne primär als Folge der Renaissance und der industriellen Revolution erklärten, zurückgewiesen werden, um die sich darin spiegelnden Projektionen europäischer Allmacht zu entlarven. Damit wird border thinking zu einer epistemologischen Position, die darauf zielt, herkömmliche Wissensformen zu transformieren, hin zu einem Wissen, das sich aus kolonialen Wunden speist und als körperliche Erfahrung lesen lässt: „Decolonial thinking precisely introduces border thinking as an epistemological position that contributes to a shift in the forms of knowing in which the world is thought from the concrete incarnated experiences of colonial difference and the wounds left" (Icaza 2017, S. 29). Inspiriert durch die feministische Philosophin María Lugones ist mit der „kolonialen Wunde“ hier nicht nur ein kultureller Code gemeint, sondern auch die unmittelbare körperliche Erfahrung, sei es von Versklavung, Rassisierung, Vergewaltigung oder Entmenschlichung (vgl. Lugones 1992, 2010). So begreift Lugones - in Auseinandersetzung mit der ebenso unterdrückten wie widerständigen Figur der Mestiza - den epistemologischen Beitrag von border thinking als ein verkörpertes Bewusstsein (vgl. ebd., S. 34), welches Dualismen und inkorporierte Verwundun- 
gen zum Ausgangspunkt eines Umdenkens nimmt, das zu einer feministischen Dekolonisierung geopolitischen Wissens und politischer Ökonomie ansetzt. ${ }^{2}$

\section{Borders und boundaries: Gendering Border Studies?}

Spätestens in den frühen 2000er Jahren erreichte die konstruktivistische Wende einen großen Teil der Border Studies (vgl. Bürkner 2017, S. 85). Grenzen wurden vermehrt als Teil alltäglicher sozialer Prozeduren und (Widerstands-)Praktiken von Akteur*innen statt als bloßer Effekt staatlicher Strukturen wahrgenommen (vgl. ebd., S. 87). Aspekte dieses Wandels wurden - analog zum Begriff des doing gender und doing difference - mit den Begriffen des bordering, rebordering und der borderization (vgl. z.B. Cassidy et al. 2018) zum Ausdruck gebracht.

Inspiriert von Forschungen aus den Cultural und Gender Studies zur Macht von ästhetischer oder symbolischer Repräsentation wurden selbst staatliche Außengrenzen nun als symbolisch hoch aufgeladene Räume gefasst, welche über Ordnungen der Differenz mitbestimmt seien. Nicht nur Kulturwissenschaftler*innen, sondern auch Geograf*innen begannen Grenzen als „soziale Praxis räumlicher Differenzherstellung“ (Houtum/Naerssen 2002, S. 126) zu definieren, als Ensembles soziokultureller Prozesse, in denen symbolische Ordnungen, Institutionen und Praktiken zusammenwirkten (vgl. Paasi 2012, S. 2304; ebenso Altink/Weedon 2010). Weiter hoben Sozialwissenschaftler"innen hervor, dass symbolische Grenzziehungen zur Härte von institutionalisierten Grenzen beitrügen und sogar integral für ihr Funktionieren seien, indem sie staatlich institutionalisierte Grenzen als natürlich und selbstverständlich erscheinen ließen (vgl. Eder 2006, S. 255f.). Ohne „Codes der sozialen Klassifikation“ und „symbolischen Verdeutlichung “ (Giesen 1993, S. 30f.) hätten Grenzen keinen Bestand. Eher selten wird dabei auf Anzaldúas frühe Arbeiten Bezug genommen, es sei denn in der feministischen Grenzforschung (aus dem angloamerikanischen Raum), die ebenfalls auf die gleichzeitige „Rhetorizität und Materialität von Grenzen “ (Flores 2019, S. 115) verweist.

Dem finnischen Geografen Anssi Paasi (2012, S. 2304) zufolge schließen „territoriale“ und „relationale“ Dimensionen von Grenzen einander nicht aus, sondern bilden vielmehr eine produktive Spannung, die das Politische als räumlichen Prozess konstituiert. Er plädiert gar für eine Überwindung der konzeptionellen Trennung zwischen „territorialen und relationalen Grenzen“, denn erst das Zusammenspiel soziokultureller, institutioneller und symbolischer Praktiken bringe Grenzen überhaupt hervor (vgl. ebd.; ebenso Cochrane/Ward 2012, S. 7). Die Humangeograf*innen Julia Lossau und Roland Lippuner (2004, S. 204) warnen hingegen vor einer Gleichsetzung von Physisch-Materiellem und Symbolischem, weil sie eine naturalisierende, „reifizierende Verräumlichung von Sozialem“ (wie dem Nationalstaat, Kulturkreis oder Kontinent) befürchten, was sie als „Raumfalle“ bezeichnen. Auch Katherine Pratt Ewing (1998) problematisiert eine Verwischung metaphorischer und materieller Grenzziehungsprozesse und verdeutlicht am Beispiel von Geschlechtergrenzen im muslimischen Kontext, dass borders und social boundaries nicht automatisch ineinander fielen, sondern unterschiedliche, sogar gegenläufige Funktionen oder Effekte haben könnten. Es sei genauer zu prüfen, wo und wie genau Grenzmetaphoriken als Teil räumlicher Ordnungen fungierten (vgl. ebd.).

2 Diese Perspektive versteht sich auch als Relativierung von radikalen antiessenzialistischen Positionen innerhalb des Feminismus, die (Geschlechts-)Identitäten vorrangig als Performanzeffekte auffassen. 
Insofern stellt eine Analyse der Wechselbeziehungen zwischen geografisch-territorialen Grenzziehungen (borders) und geschlechtlichen, religiösen oder (proto)rassistischen Formationen der Differenz (boundaries) ${ }^{3}$ für Gender und Border Studies gleichermaßen eine methodologische und transdisziplinäre Herausforderung dar (vgl. Yuval-Davis/Stoetzler 2002; Newman 2006; Jones 2009).

Exemplarisch hat etwa die historische Studie von Eithne Luibheid aus dem Jahr 2002 gezeigt, dass die US-amerikanische Grenze zwischen 1875 und 1990 entlang von geschlechtlichen, rassistischen und klassenspezifischen Grenzziehungen organisiert war. Die Kommunikationswissenschaftlerin Julia Krebtan-Hörhager stellte zudem 2019 am Beispiel von Italiens Außengrenzen einen engen Zusammenhang zwischen Praktiken einer new border culture in Europa und einem intersektionalen othering her. Aus ihrer Sicht sorge gerade die rhetorische Hervorbringung einer homogenen, spezifisch europäischen Identität und Geschichte für unüberwindbare territoriale Grenzen. (Eurozentrische) Identitätspolitiken seien dabei zentral für das Verständnis und Funktionieren von borderlands. Sie wirkten nicht nur nach außen, sondern als normalisierende, intrakulturelle Grenze auch nach innen, etwa zwischen Christ*innen und Muslim*innen, Hetero- und Homosexuellen oder Nord- und Süditaliener*innen (vgl. KrebtanHörhager 2019, S. 126).

Insofern sind Bordering-Prozesse mit Praktiken des othering eng verbunden, konstatieren auch die Geografen und Migrationsforscher Henk van Houtum und Ton van Naerssen und verweisen auf die ambivalenten Effekte dieses Zusammenhangs: Einerseits dienen Bordering-Prozesse der Herstellung einer kontrollierten räumlichen Ordnung, indem sie z.B. nationale Identität vereindeutigen und homogenisieren, andererseits sind sie mit Praktiken der Exklusion und ,Reinigung' nach innen wie außen verbunden und produzieren damit selbst neue Differenzen (vgl. Houtum/Naerssen 2002, S. 126f.). Dabei ist die Existenz einer Gruppe von Anderen, die dem Wir gegenüberstehen, dem Prozess der Grenzziehung nicht vorgängig. Vielmehr wird die Scheide zwischen ihnen durch die Bindung einer sozialen Ordnung an ein fixes Territorium erst erzeugt (vgl. ebd., S. 134).

Mit der wichtigen Rolle, die symbolisch-soziale Othering-Prozesse für die Analyse von Grenzen spielen, erweisen sich Border Studies eng mit den Erkenntnisinteressen und Methoden aus dem Bereich der Gender Studies verbunden. Dennoch fällt auf, dass viele maßgebliche Forschungen, die sich etwa mit Definitionen von Grenze befassen, ihre theoretischen Anleihen und Bezüge zu den Gender Studies nur selten explizit machen.

\section{Fazit}

Es lässt sich resümieren, dass Border und Gender Studies aus unterschiedlichen Perspektiven zu ähnlichen Problematisierungen finden, indem beide die Schnittstelle zwischen materieller und symbolischer Ordnung fokussieren und genauer zu erfassen suchen. Da diese Schnittstelle seit Langem im Zentrum der Gender Studies bzw. Differenzforschung liegt, könnten die Bor-

3 Im Anschluss an die Boundary Work-Forschung (vgl. Barth 1969; Lamont/Molnár 2002) lässt sich mit Christopher A. Bail (2008) genauer zwischen symbolischen und sozialen Grenzziehungen unterscheiden. Unter symbolic boundaries versteht er „konzeptionelle Unterscheidungen“ (ebd., S. 38f.), die Menschen in Gruppen einteilten. Social boundaries seien stärker objektivierte Formen der Differenz, die sich z.B. durch einen ungleichen Zugang zu Ressourcen artikulierten. Nur wenn symbolic boundaries große Akzeptanz fänden, könnten sie sich zu social boundaries verdichten (vgl. ebd.). 
der Studies von einer expliziteren Rezeption der interdisziplinären Genderforschung und ihren theoretisch-methodischen Reflexionen profitieren.

Die Frage, wie genau relationale und territoriale Dimensionen von Grenzziehungsprozessen miteinander verbunden sind, stellt weiterhin ein zentrales methodisch-theoretisches Desiderat wie eine produktive Herausforderung für Border und Gender Studies dar (vgl. Lossau/Lippuner 2004; ebenso Alting/Weedon 2010; Bürkner 2018). Interessant ist daher der Vorschlag des Sozialgeografen Hans-Joachim Bürkner, Untersuchungen des sozialen Imaginären stärker mit diskursanalytischen Perspektiven zusammenzubringen (vgl. Bürkner 2017, S. 93ff.). Insbesondere die Verbindung von poststrukturalistischen und diskursanalytischen Ansätzen könne die verschiedenen Dimensionen von Grenze zu erfassen helfen und das missing link zwischen individuellen und kollektiven, symbolischen wie materiellen Grenzziehungen bilden (vgl. ebd.). Grenzen ließen sich dann nicht nur als Staatsgrenzen, sondern zugleich als Formen an Körper gebundener materieller Praxis und diskursiver Bedeutungsproduktion verstehen, die Erfahrung generieren und (vergeschlechtlichte) Subjekte formieren. Entsprechend wären Grenzen weniger als Nahtstellen zwischen Territorien zu definieren, sondern als mehrdimensionale, dynamische „Bordertexturen“, die gleichermaßen aus Praktiken und Diskursen gesponnen werden (Weier et al. 2018, S. 73; vgl. auch Gerst et al. 2018).

Über die Diskussion um die Beziehung zwischen Symbolischem und Materiellem hinaus macht die Geschlechterforschung auf Fragen der Intersektionalität für Grenzziehungen aufmerksam. Folgt man intersektionalen Ansätzen der Gender Studies (vgl. Kerner 2009; Grzanka 2014b; Meyer 2019) so ist die Kategorie Geschlecht grundlegend in andere dynamische Macht- und Ungleichheitsverhältnisse wie Klasse, Alter, Religion oder Ethnizität eingebunden. Daher sind geschlechtliche Codierungen von Grenzen vor allem in ihrer jeweiligen Interrelation mit anderen Differenzkategorien zu erfassen. Durch die Einbeziehung intersektionaler Perspektiven in die Geographie könnten die engen Verbindungen zwischen Raum- und Machtproduktion genauer erfasst werden.

Diese grundlegende Intersektionalität von Differenzkategorien ist jedoch ihrerseits ohne Einbeziehung räumlicher Dimensionen in ihrer Komplexität nicht zu erfassen, wie in jüngerer Zeit expliziter herausgearbeitet wurde (vgl. Richter/Büchler 2019, S. 40; vgl. auch Carstensen-Egwuom 2016; Bruns 2016). „Oppression has not only a when, but a where“, wie Patrick R. Grzanka (2014a) anmerkte. So wird z.B. ein vergeschlechtlichter, ,rassisch“ markierter, an ein Alter gebundener Körper in einem räumlichen Gefüge materiell wie symbolisch platziert und bringt dieses seinerseits mit hervor. Es werden an den Raum gebundene bzw. diesen konstituierende Gefühle evoziert (belongings) und je bestimmte materielle wie symbolische Grenz(ziehung)en am Körper im Raum wirksam. Der Körper verweist seinerseits auf „die unausweichliche Leiblichkeit der Sprache“ im „Äußerungsraum“ zurück (Kuster 2018, S. 232). Intersektionalität als räumlich bedingte und erfahrene Kategorie zu begreifen, eröffne Feminist*innen überdies die Möglichkeit, mit der latenten Spannung zwischen multiplen individuellen Identitäten und politischen Kollektividentitäten angemessener umzugehen, wie die Geografin Gill Valentine (2007, S. 19) hervorhob.

Gloria Anzaldúa ging mit ihrem Konzept der borderlands sehr früh Figurationen von Identitätsbildungen nach, die sie als intersektional ineinandergreifend und zugleich räumlich dimensioniert entwarf:

„The actual physical borderland that I'm dealing with [...] is the Texas-U.S. Southwest/Mexican border. The psychological borderlands, the sexual borderlands and the 
spiritual borderlands are not particular to the Southwest. In fact, the Borderlands are physically present wherever two or more cultures edge each other, where people of different races occupy the same territory, where under, lower, middle and upper classes touch, where the space between two individuals shrinks with intimacy “ (Anzaldúa 1987, Vorwort).

Alter, Geschlecht und Klassendifferenzen werden so in räumliche Metaphern gekleidet und zu territorial organisierten borderlands (vgl. Pratt Ewing 1998, S. 263). Umgekehrt sind räumliche Konzepte wie „Zentrum, Peripherie, Grenze, Grenzraum und Rand“ (Smith 1996, S. 19) als wesentliche Elemente der Differenz(re)produktion zu verstehen.

Folgt man Ansätzen des Mestiza oder Border Feminism, sollten Differenzkonstruktionen indes weniger als starr verortete Achsen der Differenz denn als räumlich organisierte Zonen des Übergangs, als mobile Arrangements und Assemblagen gelesen werden (vgl. Kron 2011). Die „Konstitution politischer Subjektivitäten und die Aneignung von politischer Handlungsmacht“ würden dann eher „von der Durchkreuzung von Grenzen als einer zentralen Erfahrung her gedacht" werden denn von der Suche nach stabilen identitätsbildenden sozialen Kategorien und deren fixen „Verortungen“ (ebd., S. 218). Fragen nach der Interrelation verschiedener Differenzkategorien für räumliche Grenzziehungsprozesse zu stellen, würde zwar nach wie vor bedeuten, „die Frage der Identität“ aufzuwerfen, aber, wie Butler es formulierte, „nicht mehr nach der Identität als einer zuvor errichteten Position oder einer einheitlichen Entität, sondern als Teil einer dynamischen Landkarte der Macht, in der Identitäten gebildet und/oder ausgelöscht, eingesetzt und/oder lahmgelegt werden“ (Butler 1994, S. 134).

Insofern erweisen sich Theoretisierungsprozesse von (Geschlechter-)Differenz als relevant für künftige Transformationen des Konzepts von Grenze in den Border Studies. Aber auch umgekehrt steigert die Einbeziehung der Kategorie des (Grenz-)Raums, nicht zuletzt im Anschluss an postkoloniale Perspektiven, die Komplexität der Analysen von Differenz in den Gender Studies.

\section{Weiterführende Literatur}

Aaron, Jane/Altink, Henrice/Weedon, Chris (Hrsg.) (2010): Gendering border studies. Cardiff: University of Wales Press.

Altink, Henrice/Weedon, Chris (Hg.) (2010): „Introduction“, in: Aaron, Jane/Altink, Henrice/Weedon, Chris (Hrsg.): Gendering border studies, Cardiff: University of Wales Press, S. 1-15.

Anzaldúa, Gloria (1987): Borderlands/La Frontera: The New Mestiza. San Francisco: Sinsters/Aunt Lute.

Falk, Francesca (2011): Eine gestische Geschichte der Grenze. Wie der Liberalismus an der Grenze an seine Grenzen kommt. Paderborn: Fink.

Flores, Lisa A. (Hrsg.) (2019): Feminist Border Theories - Expanding Our Perspectives. Womens Studies in Communication 42, H. 2.

Icaza, Rosalba (2017): Decolonial feminism and global politics. Border thinking and vulnerability as a knowing otherwise. In: Woons, Marc/Weier, Sebastian (Hrsg.): Critical Epistemologies of Global Politics. Bristol: E-International Relations Publishing, S. 26-45.

\section{Literaturverzeichnis}

Aaron, Jane/Altink, Henrice/Weedon, Chris (Hrsg.) (2010): Gendering border studies. Cardiff: University of Wales Press.

Abrams, Lynn/Hunt, Karen (2000): Borders and frontiers in women's history. In: Women's History Review 9, H. 2, S. 191-200.

Akyüz, Latife (2017): Ethnicity, gender and the border economy. Living in the Turkey-Georgia borderlands, London/New York: Routledge.

Altink, Henrice/Weedon, Chris (2010): Introduction. In: Aaron, Jane/Altink, Henrice/Weedon, Chris (Hrsg.): Gendering border studies. Cardiff: University of Wales Press, S. 1-15. 
Amoore, Louise (2011): On the line: writing the geography of the virtual border. In: Political Geography 30, H. 2, S. 63-64.

Bail, Christopher A. (2008): The configuration of symbolic boundaries against immigrants in Europe. In: American Sociological Review 73, H. 1, S. 37-59.

Baker, Jennifer (2007): Reconstructing Gender and „Race“ Relations after the Frontier. In: Australian Feminist Law Journal 26, H. 1, S. 59-79.

Bandau, Anja (2004): Strategien der Autorisierung: Projektionen der Chicana bei Gloria Anzaldúa und Cherríe Moraga. Hildesheim: Olms.

Barad, Karen (2007): Meeting the Universe Halfway. Quantum Physics and the Entanglement of Matter and Meaning. Durham: Duke University Press.

Barth, Fredrik (1969): Ethnic groups and boundaries. The social organization of cultural difference. London: George Allen and Unwin.

Baumgärtner, Ingrid (2003): Biblische, mythische und fremde Frauen. Zur Konstruktion von Weiblichkeit in Text und Bild mittelalterlicher Weltkarten. In: Ertzdorff, Xenja von/Giesemann, Gerhard/Schulz, Rudolf (Hrsg.): Erkundung und Beschreibung der Welt. Zur Poetik der Reise- und Länderberichte. Amsterdam/New York: Rodopi, S. 31-86.

Beauvoir, Simone de (1949): Le deuxième sexe. Paris: Editions Gallimard.

Bell, David/Valentine, Gill (Hrsg.) (1995): Mapping desire. Geographies of sexualities. London/New York: Routledge.

Bennett, Jane (2004): The Force of Things. Steps toward an Ecology of Matter. In: Political Theory 32, H. 3, S. 347-372.

Bhabha, Homi K. (2007): Die Verortung der Kultur. Tübingen: Stauffenburg.

Blunt, Alison/Rose, Gillian (1994): Writing women and space. Colonial and postcolonial geographies. New York: Guilford Press.

Bourdieu, Pierre (1997): Die männliche Herrschaft. In: Dölling, Irene/Krais, Beate (Hrsg.): Ein alltägliches Spiel. Geschlechterkonstruktion in der sozialen Praxis. Frankfurt/M.: Suhrkamp, S. 153-217.

Braun, Christina von/Mathes, Bettina (2007): Verschleierte Wirklichkeit. Die Frau, der Islam und der Westen. Berlin: Aufbau.

Braun, Christina von/Stephan, Inge (Hrsg.) (2013): Gender@Wissen: Ein Handbuch der Gender-Theorien. 3. Aufl., Wien/Köln/Weimar: Böhlau.

Breger, Claudia (2010): Gender Studies. In: Esslinger, Eva/Schlechtriemen, Tobias/Schweitzer, Doris/Zons, Alexander (Hrsg.): Die Figur des Dritten. Ein kulturwissenschaftliches Paradigma. Frankfurt/M.: Suhrkamp, S. 35-48.

Browne, Kath/Lim, Jason/Brown, Gavin (Hrsg.) (2009): Geographies of sexualities. Theory, practices and politics. Farnham: Ashgate.

Bruns, Claudia (2009): Europas Grenzdiskurse seit der Antike - Interrelationen zwischen kartographischem Raum, mythologischer Figur und europäischer ,Identität‘. In: Gehler, Michael/Pudlat, Andreas (Hrsg.): Grenzen in Europa. Hildesheim: Ohlms, S. 17-64.

Bruns, Claudia (2015): Die Grenzen des „Volkskörpers“. Interrelationen zwischen „Rasse“, Raum und Geschlecht in NS-Geopolitik und Kunst. In: Feministische Studien 33, H. 2, S. 177-196.

Bruns, Claudia (2016): „Rasse“ und Raum. Überlegungen zu einer komplexen Relation. In: Dies. (Hrsg.): „Rasse“ und Raum. Topologien zwischen Kolonial-, Geo- und Biopolitik. Geschichte, Kunst, Erinnerung. Wiesbaden: Reichert, S. 1-44.

Bruns, Claudia (i.E.): Europas Grenzen: Karten, Körper, Kollektive seit der Antike. Wien/Köln/Weimar: Böhlau.

Bürkner, Hans-Joachim (2017): Bordering, borderscapes, imaginaries. From constructivist to post-structural perspectives. In: Opiłowska, Elżbieta/Kurcz, Zbigniew/Roose, Jochen (Hrsg.): Advances in European Borderlands Studies. Baden-Baden: Nomos, S. 85-108.

Butler, Judith (1990): Gender Trouble. Feminism and the Subversion of Identity. New York/London: Routledge.

Butler, Judith (1994): Phantasmatische Identifizierung und die Annahme des Geschlechts. In: Pühl, Katharina (Hrsg.): Geschlechterverhältnisse und Politik. Frankfurt/M.: Suhrkamp, S. 101-138.

Butler, Judith (1997): Das Ende der Geschlechterdifferenz? In: Huber, Jörg./Heller, Martin (Hrsg): Interventionen 6. Konturen des Unentschiedenen. Basel/Frankfurt/M.: Stroemfeld, S. 25-43.

Butler, Judith (2010): Raster des Krieges. Warum wir nicht jedes Leid beklagen. Frankfurt/M./New York: Campus.

Cantú, Lionel (2009): The sexuality of migration: Border crossings and Mexican immigrant men 5. New York/London: New York University Press.

Carstensen-Egwuom, Inken (2018): Intersektionalität und Transnationalismus zusammen denken. Eine intersektionale Perspektive auf transnationale soziale Positionierungen nigerianischer Migranten in Bre- 
men. Diss. Online Open Access, Flensburg: Europa-Universität Flensburg, www.zhb-flensburg.de/?id=2 $6355,1.9 .2020$.

Cassidy, Kathryn/Yuval-Davis, Nira/Wemyss, Georgie (2018): Debordering and everyday (re) bordering in and of Dover: Post-borderland borderscapes. In: Political Geography 66, S. 171-179.

Castañeda, Antonia (Hrsg.) (2007): Gender on the borderlands. The Frontiers Reader. Lincoln: University of Nebraska Press.

Castillo, Debra A./Tabuenca Córdoba, María Soccorro (2002): Border Women: Writing from La Frontera. Minneapolis: University of Minnesota Press.

Chaudhuri, Nupur (1998): Nation, Empire, Colony: Historicizing Gender and Race. Bloomington: Indiana University Press.

Clancy-Smith, Julia (2005): Women, Gender and Migration along a Mediterranean Frontier: Pre-Colonial Tunisia, c. 1815-1870. In: Gender \& History 17, H. 1, S. 62-92.

Cochrane, Allan/Ward, Kevin (2012): Researching the geographies of policy mobility: confronting the methodological challenges. In: Environment and Planning A 44, S. 5-12.

Combahee River Collective (1982): A Black Feminist Statement [1977]. In: Hull, Gloria/Scott, Patricia/Smith, Barbara (Hrsg.): All the Women are White, All the Blacks are Men, But Some of Us Are Brave. New York: The Feminist Press, S. 13-22.

Côté-Boucher, Karine (2020): Grenzbrüche: Geschlecht, Generation und Technologie an vorderster Front. New York/London: Routledge.

Cotten, Trystan T. (Hrsg.) (2012): Transgender migrations: The bodies, borders, and politics of transition. New York/London: Routledge.

Crawford, Kerry F. (2017): Wartime sexual violence. From silence to condemnation of a weapon of war. Washington: Georgetown University Press.

Cusack, Tricia/Bhreathnach-Lynch, Síghle (Hrsg.) (2018): Art, nation and gender. Ethnic landscapes, myths and mother-figures. New York: Routledge.

Del Re, Emanuela C. (2018): Gendering the European borders: The role of female migrants and refugees. In: Shekhawat, Seema/Del Re, Emanuela C. (Hrsg.): Women and borders: refugees, migrants and communities. London/New York: Tauris, S. 21-38.

Deleuze, Gilles/Guattari, Félix (1992): Tausend Plateaus. Kapitalismus und Schizophrenie. Berlin: Merve.

Dietrich, Anette (2015): Weiße Weiblichkeiten: Konstruktionen von „Rasse“ und Geschlecht im deutschen Kolonialismus. Bielefeld: transcript.

Dietze, Gabriele (2006): Critical Whiteness Theory und Kritischer Okzidentalismus. Zwei Figuren hegemonialer Selbstreflexion. In: Tißberger, Martina/Dietze, Gabriele/Hrzán, Daniela/Husmann-Kastein, Jana (Hrsg.): Weiß - Weißsein - Whiteness. Kritische Studien zu Gender und Rassismus. Frankfurt/M.: Peter Lang, S. 219-248.

Dietze, Gabriele (2019): Sexueller Exzeptionalismus: Überlegenheitsnarrative in Migrationsabwehr und Rechtspopulismus. Bielefeld: transcript.

Domosh, Mona/Seager, Joni (2001): Putting Women in Place: Feminist Geographers make sense of the world. New York: Guilford Press.

Eder, Klaus (2006): Europe's borders. The narrative construction of the boundaries of Europe. In: European Journal of Social Theory 9, H. 2, S. 255-271.

Flores, Lisa A. (2019): At the Intersections: Feminist Border Theory. In: Women's Studies in Communication 42, H. 2: Feminist Border Theories - Expanding Our Perspectives, S. 113-115.

Fredrich, Bettina (2012): Verorten - verkörpern - verunsichern. Eine Geschlechtergeografie der Schweizer Sicherheits- und Friedenspolitik. Bielefeld: transcript.

Funk, Julika (1998): Maske - Grenze - Geschlecht. Bemerkungen zur Lesbarkeit von Geschlechterdifferenz im kulturellen Gedächtnis der Moderne. In: Deutsche Vierteljahrsschrift für Literaturwissenschaft und Geistesgeschichte 72, H. 1, S. 193-212.

Ganser, Alexandra (2013): Review: Gendering Border Studies by Jane Aaron, Henrice Altink and Chris Weedon. In: Amerikastudien/American Studies 58, H. 3, S. 509-514.

Gerst, Dominik/Klessmann, Maria /Krämer, Hannes/Sienknecht, Mitja/Ulrich, Peter (2018): Komplexe Grenzen. Aktuelle Perspektiven der Grenzforschung. In: Berliner Debatte Initial 29, H. 1, S. 3-11.

Giesen, Bernhard (1993): Die Intellektuellen und die Nation. Eine deutsche Achsenzeit. Frankfurt/M.: Suhrkamp.

Gilroy, Paul (1993): The Black Atlantic. Modernity and double consciousness. Harvard: University Press.

Gradinari, Irina/Li, Yumin/Naumann, Myriam (Hrsg.) (2020): Europas Außengrenzen. Interrelationen von Raum, Geschlecht und „Rasse“. Bielefeld: transcript.

Grzanka, Patrick R. (2014a): Space, Place, Communities, and Geographies. In: Ders.: Intersectionality (Hrsg.): Foundations and frontiers. New York/London: Routledge, S. 99-106. 
Grzanka, Patrick R. (Hrsg.) (2014b): Intersectionality: Foundations and frontiers. New York/London: Routledge.

Haraway, Donna (1995a): Die Neuerfindung der Natur: Primaten, Cyborgs und Frauen. Hrsg. v. Carmen Hammer und Immanuel Stieß. Frankfurt/M.: Campus.

Haraway, Donna (1995b): Situiertes Wissen. Die Wissenschaftsfrage im Feminismus und das Privileg einer partialen Perspektive. In: Dies.: Die Neuerfindung der Natur. Primaten, Cyborgs und Frauen. Frankfurt/M.: Campus.

Harvey, Elizabeth (2010): „Der Osten braucht Dich!“ Frauen und nationalsozialistische Germanisierungspolitik. 2. Aufl., Hamburg: Hamburger Edition.

Hall, Donald E./Jagose, Annamarie (Hrsg.) (2012): The Routledge Queer Studies Reader. New York/London: Routledge.

Hannah, Matthew G. (2005): Virility and Violation in the US „War on Terrorism“. In: Nelson, Lise/Seager, Joni (Hrsg.): A Companion to Feminist Geography. Malden/Oxford: Blackwell, S. 550-564.

Harley, John Brian (1988): Maps, knowledege, and power. In: Cosgrove, Denis E./Daniels, Stephen (Hrsg.): The Iconography of Landscape. Cambridge/New York: Cambridge University Press, S. 277312.

Hausen, Karin/Heide Wunder (Hrsg.) (1992): Frauengeschichte - Geschlechtergeschichte. Bd. 1. Frankfurt/M.: Campus.

Heide, Markus (2004): Grenzüberschreibungen: Chicano/a-Erzählliteratur und die Inszenierung von Kulturkontakt. Heidelberg: Universitätsverlag Winter.

Hernández, Leandra Hinojosa (2019): Feminist Approaches to Border Studies and Gender Violence: Family Separation as Reproductive Injustice. In: Women's Studies in Communication 42, H. 2, S. 130-134.

Hess, Sabine/Neuhauser, Johanna/Schwenken, Helen (2016): Unter- oder überbelichtet: Die Kategorie Geschlecht in medialen und wissenschaftlichen Diskursen zu Flucht. In: Hess, Sabine/Kasparek, Bernd/ Kron, Stefanie/Rodatz, Mathias /Schwertl, Maria/Sontowski, Simon (Hrsg.): Der lange Sommer der Migration. Grenzregime III. Berlin, S. 176-195.

Hogan, Maureen P./Pursell, Timothy (2008): The „Real Alaskan“. Nostalgia and Rural Masculinity in the „Last Frontier“. In: Men and Masculinities 11, H. 1, S. 63-85.

Hoppe, Katharina/Lemke, Thomas (2015): Die Macht der Materie. Grundlagen und Grenzen des agentiellen Realismus von Karen Barad. In: Soziale Welt, Bd. 66, S. 261-279.

Houtum, Henk van/Naerssen, Ton van (2002): Bordering, Ordering, and Othering. In: Journal of Economic and Social Geography (TESG) 93, H. 2, S. 125-136.

Johnson, E. Patrick/Henderson, Mae G. (Hrsg.) (2005): Black Queer Studies: A Critical Anthology. Durham: Duke University Press.

Jones, Adam (2002): Gender and genocide in Rwanda. In: Journal of Genocide Research 4, H. 1, 65-94.

Jones, Reece (2009): Categories, borders and boundaries. In: Progress in Human Geography 33, H. 2, S. $174-189$.

Keating, Ana Louise (2016): EntreMundos/AmongWorlds: Neue Perspektiven auf Gloria E. Anzaldúa. Wiesbaden: Springer VS.

Kerner, Ina (2009b): Differenzen und Macht. Zur Anatomie von Rassismus und Sexismus. Frankfurt/M.: Campus.

Krebtan-Hörhager, Julia (2019): Intersectional othering and new border cultures. Lessons from Italy. In: Women's Studies in Communication 44, H. 2, S. 125-129.

Klinger, Cornelia (1995): Beredtes Schweigen und verschwiegenes Sprechen: Genus im Diskurs der Philosophie. In: Bußmann, Hadumod/Hof, Renate (Hrsg.): Genus - zur Geschlechterdifferenz in den Kulturwissenschaften. Stuttgart: Kröner, S. 34-59.

Knapp, Gudrun-Axeli (1988): Die vergessene Differenz. Feministische Studien. Zeitschrift für interdisziplinäre Frauen-und Geschlechterforschung 6, H. 1, S. 12-31.

Koschorke, Albrecht/Lüdemann, Susanne/Frank, Thomas/Matala Mazza, Ethel de (2007): Der fiktive Staat. Konstruktionen des politischen Körpers in der Geschichte Europas. Frankfurt /M.: Fischer.

Koskela, Hille (2012): „You shouldn't wear that body.“ The problematic of surveillance and gender. In: Ball, Kirstie/Lyon, David /Haggerty, Kevin D. (Hrsg.): Routledge-Handbuch für Überwachungsstudien. New York/London: Routledge, S. 49-56.

Krewani, Angela (2000): Geschlecht und Raum. Überlegungen zu Travestie, Crossdressing und GenderBending als räumliche Kategorien. In: Becker, Barbara/Schneider, Irmela (Hrsg.): Was vom Körper übrig bleibt. Körperlichkeit - Identität - Medien. Frankfurt/M.: Campus, S. 197-208.

Kron, Stefanie (2011): Intersektionalität oder borderland als Methode? Zur Analyse politischer Subjektivitäten in Grenzräumen. In: Hesse, Sabine/Langreiter, Nikola/Timm, Elisabeth (Hrsg.): Intersektionalität revisited. Bielefeld: transcript, S. 197-221. 
Kuster, Brigitta (2018): Grenze filmen. Eine kulturwissenschaftliche Analyse audiovisueller Produktionen an den Grenzen Europas. Bielefeld: transcript.

Lamont, Michèle/Molnár, Virág (2002): The Study of boundaries in the social sciences. In: Annual Review of Sociology 28, S. 167-195.

Lau, Lasse/Arsanios, Mirene/Zúniga-González/Kryger, Mathias/Mismar, Omar (2014): Queer Geographies. Roskilde: Museet for Samtidskunst.

Lewis, Reina/Mills, Sara (2003): Feminist Postcolonial Theory. A Reader. Edinburgh: University Press.

Le Breton, Maritza (1998): Die Feminisierung der Migration. Eine Analyse im Kontext neoliberaler Arbeits- und Aufenthaltsverhältnisse. In: Agarwal, Bina (Hrsg.): Globalisierung aus Frauensicht. Bilanzen und Visionen. Berlin: Dietz, S. 112-134.

List, Elisabeth (1997): Das lebendige Selbst. Leiblichkeit, Subjektivität und Geschlecht. In: Stoller, Silvia/Vetter, Helmuth (Hrsg.): Phänomenologie und Geschlechterdifferenz. Wien: WUV, S. 292-318.

Lobasz, Jennifer K (2009): Beyond Border Security: Feminist Approaches to Human Trafficking. In: Security Studies 18, H. 2, S. 319-344.

Longhurst, Robyn (2001): Bodies: exploring fluid boundaries. New York/London: Routledge.

Lossau, Julia (2009): Spatial turn, cultural turn und Kulturgeographie. In: Csáky, Moritz/Leitgeb, Christoph (Hrsg.): Kommunikation - Gedächtnis - Raum. Kulturwissenschaften nach dem „Spatial Turn“. Bielefeld: transcript, S. 29-44.

Lossau, Julia/Lippuner, Roland (2004): Geographie und spatial turn. In: Erdkunde, Bd. 58, H. 3, S. $201-$ 211.

Lugones, María (1992): On borderlands/La frontera: An interpretive essay. In: Hypatia 7, H. 4, S. 31-37.

Lugones, María (2010): Towards a Decolonial Feminism. In: Hypathia 25, H. 4, S. 742-759.

Luibhâeid, Eithne (2002): Entry denied. Controlling sexuality at the border. Minneapolis: University of Minnesota Press.

Lutz, Helma (2008): Migrations- und Geschlechterforschung: Zur Genese einer komplizierten Beziehung. In: Becker, Ruth/Kortendiek, Beate (Hrsg.): Handbuch Frauen- und Geschlechterforschung. Wiesbaden: VS, S. 565-573.

Magnet, Shoshana (2011): Wenn die Biometrie versagt: Geschlecht, Rasse und die Technologie der Identität. Durham: Duke University Press.

Mamozei, Martha (1992): Frauen und Kolonialismus - Täterinnen und Opfer. In: Foitzik, Andreas/Leiprecht, Rudolf/Marvakis, Athanasios/Seid, Uwe (Hrsg.): „Ein Herrenvolk von Untertanen“. Rassismus Nationalismus - Sexismus. Duisburg: DISS, S. 125-142.

[Mandeville, John] (2005): The travels of Sir John Mandeville. Übersetzt und eingeleitet durch Charles William Reuben Dutton Moseley. 2. Aufl., London/New York: Penguin Books.

Marston, Sallie A./Jones, John Paul/Woodward, Keith (2005): Human Geography without Scale. In: Transactions of the Institute of British Geographers 30, H. 4, S. 416-432.

Massey, Doreen (1999): Spaces of Politics. In: Dies./Allen, John/Sarre, Phil (Hrsg.): Human Geography Today. Cambridge: Polity Press, S. 279-294.

Mateos-Aparicio, Ángel (2007): Trespassers of Body Boundaries: The Cyborg and Construction of a Postgendered Posthuman Identity. In: Manzanas, Ana María (Hrsg.): Border Transits. Literature and Culture across the Line. Amsterdam/New York: Rodopi, S. 243-276.

Mertlitsch, Kirstin (2016): Sister - Cyborg - Drags. Das Denken in Begriffspersonen der Gender Studies. Bielefeld: transcript.

Meyer, Katrin (2019): Theorien der Intersektionalität zur Einführung. Hamburg: Junius.

Michels, Stefanie (2009): Weiße Frauen in Afrika: Grenzwächterinnen und Grenzüberschreiterinnen im (post)kolonialen Haushalt. In: Ariadne 56, „Das schöne Wunderland in der Ferne“. Geschlechterdimensionen des deutschen Kolonialismus, S. 24-31.

Morokvasic, Mirjana (2006): Crossing Borders and Shifting Boundaries of Belonging in post-Wall Europe. A gender lens. In: Berthoin Antal, Ariane/Quack, Sigrid (Hrsg.): Grenzüberschreitungen - Grenzziehungen. Implikationen für Innovationa und Identität. Berlin: Sigma, S. 47-72.

Newman, David (2006): Borders and bordering: towards an interdisciplinary dialoge. In: European Journal of Social Theory 9, H. 20, S. 171-186.

Nieden, Susanne zur (2002): Erotische Fraternisierung. Der Mythos von der schnellen Kapitulation der deutschen Frauen im Mai 1945. In: Hagemann, Karen/ Schüler-Springorum, Stefanie (Hrsg.): HeimatFront. Militär und Geschlechterverhältnisse im Zeitalter der Weltkriege. Frankfurt/M./New York: Campus, S. 313-326.

Paasi, Anssi (1996): Inclusion, exclusion and territorial identities. The meanings of boundaries in the globalizing geopolitcal landscape. In: Nordisk Samhällsgeografisk Tidskrift 23, S. 6-23.

Paasi, Anssi (2012): Border studies reanimated: going beyond the territorial/relational divide. In: Environment and Planning A: Economy and Space 44, H. 10, S. 2303-2309. 
Pápay, Gyula (2005): Die Beziehung von Kartographie, allgemeiner Bildwissenschaft und Semiotik. In: Sachs-Hombach, Klaus (Hrsg.): Bildwissenschaft zwischen Reflexion und Anwendung. Köln: Herbert von Halem, S. 86-100.

Parr, Rolf (2008): Liminale und andere Übergänge. Theoretische Modellierungen von Grenzzonen, Normalitätsspektren, Schwellen, Übergängen und Zwischenräumen in Literatur-und Kulturwissenschaft. In: Geisenhanslüke, Achim/Mein, Georg (Hrsg.): Schriftkultur und Schwellenkunde. Bielefeld: transcript, S. 11-63.

Penttinen, Elina (2007): Globalisierung, Prostitution und Sexhandel: Körperpolitik. New York/London: Routledge.

Perales, Monica (2013): On Borderlands/La Frontera: Gloria Anzaldúa and Twenty-Five Years of Research on Gender in the Borderlands. In: Journal of Women's History 25, H. 4, S. 163-173.

Pieper, Marianne/Kuster, Brigitta/Tsianos, Vassilis (2014): Transnationale Akteur_innen der Migration: Gender-Netzwerke-Assemblagen: Methodologische und methodische Überlegungen im Zeichen einer neuen Ontologie. In: Gruhlich, Julia/Riegraf, Birgit (Hrsg.): Geschlecht und transnationale Räume. Feministische Perspektiven auf neue Ein- und Ausschlüsse. Münster: Westfälisches Dampfboot, S. 227249.

Pratt, Mary Louise (1991): Arts of the contact zone. In: Profession. New York: Modern Language Association, S. 33-40.

Pratt Ewing, Katherine (1998): Crossing borders and transgressing boundaries: Metaphors for negotiating multiple identities. In: Ethos 26, H. 2, S. 262-267.

Reichert, Ramón (2011): Das Geschlecht der Grenze. Genderrepräsentation von der Berliner Mauer bis zur EU-Außengrenze. In: Dennerlein, Bettina/Frietsch, Elke (Hrsg.): Identitäten in Bewegung. Migration im Film. Bielefeld: transcript, S. 35-56.

Renger, Almut-Barbara/Ißler, Roland Alexander (Hrsg.) (2009): Europa - Stier und Sternenkranz. Von der Union mit Zeus zum Staatenverbund. Göttingen: V \& R Unipress.

Richter, Marina/Büchler, Bettina (2019): Here-and-now: spatialising intersectionality. In: Raibaud, Yves/ Marius, Kamala (Hrsg.): Genre et Construction de la Géographie. Pessac: Maison des Sciences de L'Homme d'Aquitaine, S. 39-52.

Riyal, AL. Mohamed (2019): Post-colonialism and Feminism. In: Asian Social Science 15, H. 11, S. 83-88.

Römhild, Regina (2009): Aus der Perspektive der Migration. Die Kosmopolitisierung Europas. In: Binder, Jana/Hess, Sabine/Moser, Johannes (Hrsg.): No integration?! Kulturwissenschaftliche Beiträge zur Integrationsdebatte in Europa. Bielefeld: transcript, S. 225-238.

Runte, Anette (2006): Über die Grenze: zur Kulturpoetik der Geschlechter in Literatur und Kunst. Bielefeld: transcript.

Rutherford, Jonathan/Bhabha, Homi K. (1990): Interview with Homi Bhabha. The third space. In: Rutherford, Jonathan (Hrsg.): Identity. Community, culture, difference. London: Lawrence \& Wishart, S. 207-221.

Said, Edward W. (1978/2009): Orientalismus. Aus dem Englischen übers. von Hans Günter Holl. Frankfurt/M.: Fischer.

Saldívar-Hull, Sonia (Hrsg.) (2000): Feminism on the border. Chicana gender politics and literature. Berkeley u.a.: University of California Press.

Sarasin, Philipp (2004): Abu Ghraib, „Terrorist Vermin“ und der utopische Körper der Nation. In: Hasselmann, Kristiane/Schmidt, Sandra/Zumbusch, Cornelia (Hrsg.): Utopische Körper. Visionen künftiger Körper in Geschichte, Kunst und Gesellschaft. München: Fink, S. 167-182.

Saul, Nicholas/Möbus, Frank (1999): Zur Einführung: Schwelle - Metapher und Denkfigur. In: Saul, Nicholas/Steuer, Daniel/Möbius, Frank/Illner, Birgit (Hrsg.): Schwellen. Germanistische Erkundungen einer Metapher. Würzburg: Königshausen \& Neumann, S. 9-15.

Scheibelhofer, Paul (2011): Intersektionalität, Männlichkeit und Migration - Wege zur Analyse eines komplizierten Verhältnisses. In: Hess, Sabine/Langreiter, Nikola/Timm, Elisabeth (Hrsg.): Intersektionalität revisited. Bielefeld: transcript, S. 149-172.

Schlögel, Karl (2007): Räume und Geschichte. In: Günzel, Stephan (Hrsg.): Topologie. Zur Raumbeschreibung in den Kultur- und Medienwissenschaften. Bielefeld: transcript, S. 33-51.

Schmale, Wolfgang (2000): Europa - die weibliche Form. In: l'homme. Europäische Zeitschrift für Feministische Geschichtswissenschaft 11, H. 2, S. 211-233.

Scott, Joan (1986): Gender: A Useful Category of Historical Analysis. In: The American Historical Review 91, H. 5, S. 1053-1075.

Segrave, Marie/Pickering, Sharon (2012): Sexhandel. New York/London: Routledge.

Sehnbruch, Lucia (2019): Re/Präsentation von Flucht und Migration durch Bildschirmmedien. In: Arslan, Emre/Bozay, Kemal (Hrsg.): Symbolische Ordnung und Flüchtlingsbewegungen in der Einwanderungsgesellschaft. Wiesbaden: Springer VS, S. 249-272. 
Sieber Cornelia (2004): Gloria Anzalduas „autohistoria“ - Das Konzept einer hybriden Identität in Borderlands/La Frontera. In: De Toro, Alfonso/Gronemann, Claudia (Hrsg.): Autobiographie revisited. Theorie und Praxis neuer autobiographischer Diskurse in der französischen, spanischen und lateinamerikanischen Literatur. Hildesheim u.a.: Olms, S. 207-224.

Slotkin, Richard (1998): Gunfighter nation: The myth of the frontier in twentieth-century America. Norman: University of Oklahoma Press.

Smith, Susan J. (1996): Bordering on identity. In: Scotlands 3, H. 1, S. 18-31.

Smith, Barbara (Hrsg.) (2000): Home girls: A black feminist anthology. 2. Aufl., New Brunswick/New Jersey/London: Rutgers University Press.

Somerville, Margaret/Perkins, Tony (2003): Border work in the contact zone: Thinking indigenous/non-indigenous collaboration spatially. In: Journal of Intercultural Studies 24, H. 3, S. 253-266.

Spindler, Susanne (2011): Feminisierung von Migration - Formen und Folgen weiblicher Wanderungsprozesse. In: Hentges, Gudrun/Platzer, Hans-Wolfgang (Hrsg.): Europa - quo vadis? Ausgewählte Problemfelder der europäischen Integrationspolitik. Wiesbaden: VS, S. 171-186.

Spivak, Gayatri C. (1988): Can the Subaltern speak. In: Nelson, Cary/Grossberg, Lawrence (Hrsg.): Marxism and the Interpretation of Culture. Urbana: University of Illinois Press, S. 271-313.

Strüver, Anke (2005): Macht - Körper - Wissen - Raum? Ansätze für eine Geographie der Differenzen. Wien: Institut für Geographie und Regionalforschung der Universität Wien.

Ticktin, Miriam (2008): Sexual Violence as the Language of Border Control: Where French Feminist and Anti-immigrant Rhetoric Meet. In: Signs 33, H. 4, S. 863-889.

Toksöz, Gülay/Ünlütürk Ulutaş, Çağla (2012): Is Migration Feminized? A Gender and Ethnicity Based Review of the Literature on Irregular Migration to Turkey. In: Paçaci Elitok, Seçil/Straubhaar, Thomas (Hrsg.): Turkey, Migration and the EU: Potentials, challenges and opportunities, 2012, 5. Jg., S. 85-112.

Turner, Frederick J. (1920): The Frontier in American History. New York: Henry Holt.

Tyszler, Elsa (2019): From controlling mobilities to control over women's bodies: gendered effects of EU border externalization in Marocco. In: Comparative Migration Studies 7, H. 25, S. 1-20.

Valentine, Gill (2007): Theorizing and Researching Intersectionality: A Challenge for Feminist Geography. In: The Professional Geographer 59, S. 10-21.

Vuolajärvi, Niina (2015): Precarious intimacies: the European border régime and migrant sex work. In: Journal of Ethnic and Migration Studies 45, H. 7, S. 1090-1107.

Waniek, Eva/Stoller, Silvia (Hrsg.) (2001): Verhandlungen des Geschlechts: Zur Konstruktivismusdebatte in der Gender-Theorie. Wien: Turia+ Kant.

Wastl-Walter, Doris (2010): Gender-Geographien. Geschlecht und Raum als soziale Konstruktionen. Stuttgart: Steiner.

Weier, Sebastian/Fellner, Astrid M./Frenk, Joachim/Kazmaier, Daniel/Michely, Eva/Vatter, Christoph/Weiershausen, Romana/Wille, Christian (2018): Bordertexturen als transdisziplinärer Ansatz zur Untersuchung von Grenzen. Ein Werkstattbericht. In: Berliner Debatte Initial 29, H. 1, S. 73-83.

Wenk, Silke (1996): Versteinerte Weiblichkeit. Allegorien in der Skulptur der Moderne. Wien/Köln/ Weimar: Böhlau.

Werner, Michael/Zimmermann, Bénédicte (2003): Penser l'histoire croisée: entre empirie et réflexivité. In: Annales. Histoire, sciences sociales 58, H. 1, S. 7-36.

Wintle, Michael J. (2009): The image of Europe. Visualizing Europe in cartography and iconography throughout the ages. Cambridge: Cambridge University Press.

Women and Geography Study Group (1997): Feminist Geographies: Explorations in Diversity and Difference. Essex: Longman.

Wright, Melissa W. (1998): Maquiladora Mestizas and a feminist border politics: revisiting Anzaldúa. In: Hypatia 13, H. 3, S. 114-131.

Wright, Melissa W. (2011): Necropolitics, narcopolitics, and femicide: Gendered violence on the MexicoUS border. In: Signs: Journal of Women in Culture and Society 36, H. 3, S. 707-731.

Yuval-Davis, Nira (2001): Geschlecht und Nation. Emmendingen: Die Brotsuppe.

Yuval-Davis, Nira/Stoetzler, Marcel (2002): Imagined Boundaries and Borders: A Gendered Gaze. In: European Journal of Women's Studies 9, H. 3, S. 329-344. 